\title{
IMPACTO DE LA EVOLUCION DEL MERCADO DEL ACEITE DE ARGÁN SOBRE LA ARGANERÍA DE MARRUECOS
}

\section{THE IMPACT OF MARKET EVOLUTION OF ARGAN OIL ON MOROCCAN ARGANERAIE}

Hassan FAOUZI ${ }^{1}$

\author{
Oficina de Investigación e Ingeniería, Agadir, Marruecos
}

\begin{abstract}
RESUMEN
El árbol de argán (Argania spinosa (L) Skeels) es un árbol endémico de Marruecos, utilizado para la alimentación de las personas y los animales. El aceite de argán es la producción más conocida de la arganería y la más valorada gracias a su atractivo creciente, muy apreciado por las industrias de cosméticos de lujo, los laboratorios farmacéuticos y los grandes cocineros, es ciertamente uno de los recursos locales de las regiones del sur, que ha conocido estos últimos años el ascenso comercial más fulgurante; pero, en el sur-oeste de Marruecos, las poblaciones de la Argania spinosa están en constante disminución y su regeneración está comprometida. Durante una quincena de años se han hecho esfuerzos para definir las condiciones de un desarrollo verdaderamente sostenible de la arganería marroquí. El aceite de argán, generado a partir de la almendra oleaginosa de los frutos de argán, es ciertamente la producción sobre la que podría basarse un proyecto de desarrollo socio-económico. Con base en su éxito, en tan solo una década el aceite de argán ha inundado el mercado de los cosméticos de Europa y América del norte. Sin embargo, algunos años más tarde se ha constatado que el desarrollo comercial del aceite de argán, no ha tenido ningún efecto real sobre la conservación de los bosques de Argania, poniendo así en peligro la presencia de este árbol y su ecosistema ya muy frágil.
\end{abstract}

Palabras clave: Argán, arganería, aceite de argán, desforestación, desarrollo, Marruecos

1 Hassan FAOUZI, Doctor en geografía, ordenación del territorio y el paisaje. Geofao, Oficina de Investigación e Ingeniería, Agadir, Marruecos. Correo electrónico: faouzi@uiz.ac.ma

Fecha de recepción: 10 de marzo de 2015

Fecha de aceptación: 1 de junio de 2015 


\begin{abstract}
The argan tree (Argania spinosa (L) Skeels) is an endemic tree from Morocco that is used to feed people and animals. Argan oil is the most famous and most valued product of the argan tree due to its growing attractiveness. Prized by the industries of luxury cosmetic products and pharmaceutical products and by chefs, it is certainly one of the local resources of southern regions which has had a remarkably commercial growth in the last years. But in the southwestern region of Morocco, argan stands are steadily decreasing and regeneration is thus compromised. For fifteen years, efforts have been made to define the conditions for a truly sustainable development of the Moroccan arganeraies. Argan oil, produced from the edible oil from the argan fruit (affiache), is certainly the production which could be the basis for a socio-economic development project. Based on its success, argan oil has flooded the market of cosmetic products in Europe and North America in just a decade. However, a few years later, it has been verified that the commercial development of the "argan oil" industry has not had real effects on the preservation of argan forests, thus threatening this tree and its already weakened ecosystem.
\end{abstract}

Keywords: Argan, aganeraie, argan oil, deforestation, development, Morocco

\title{
Introducción
}

El aceite de argán es, actualmente, uno de los aceites más caros del mundo. En 1990, con el descubrimiento de sus virtudes culinarias, cosméticas y medicinales, se ha convertido en un producto muy interesante. Las agencias de desarrollo, organizaciones no gubernamentales y cooperativas de aceite de argán, han jugado un papel importante en este auge del mercado, con el objetivo de mejorar los medios de vida locales y la conservación de las Arganias. La finalidad de este artículo es estudiar el impacto del crecimiento del mercado de argán, que registró un aumento en los precios de los productos de la arganería.

\section{Metodología}

Este estudio está enmarcado en un diseño metodológico de tipo descriptivo. Para ello, diferentes enfoques científicos se han movilizado en esta investigación: recolección de datos ambientales y estadísticos, una serie de fotografías de satélite y observaciones de campo. También, hemos utilizado otros métodos cualitativos para la recolección de datos. La principal técnica utilizada consiste en la observación directa, encuestas y la entrevista en profundidad con la población y los actores locales.

\section{El argán y su importancia}

El árbol de argán (Argania spinosa (L). Skeels) (figura 1) que Emberger (1938), califica como "árbol de hierro" y “bienestar", es una especie frutal del bosque, de usos múltiples que forma una región forestal característica en el sur-oeste de Marruecos, donde ocupa 830.000 hectáreas (M'Hirit et al, 
1998) (figura 1). Cada parte del árbol es útil y es una fuente de ingresos o alimentos para el usuario. Gracias a sus raíces profundas, el argán es una excelente barrera contra la desertización, bajo su sombra viven una fauna y flora, cuya presencia es fundamental para el equilibrio ecológico de esta región del suroeste de Marruecos. En estas zonas áridas, 2 millones de personas se ven afectadas por la explotación de sistemas agro-forestales tradicionales basados en la arganería; pero debido a la sobrexplotación, este entorno frágil se ve amenazado, casi la mitad de los bosques de Arganias han desaparecido en el transcurso del siglo XX; esta regresión es debida a la explotación de los recursos forestales (carbón, pastoreo, producción agrícola, vareo, etc.) (Faouzi, 2003). Por eso, en los últimos quince años, se han hecho esfuerzos para definir las condiciones de un desarrollo verdaderamente sostenible.

Figura 1. El árbol de argán, endémico de Marruecos, es la especie más notable del norte de África, tanto por el interés botánico y bioecológico como por su valor social

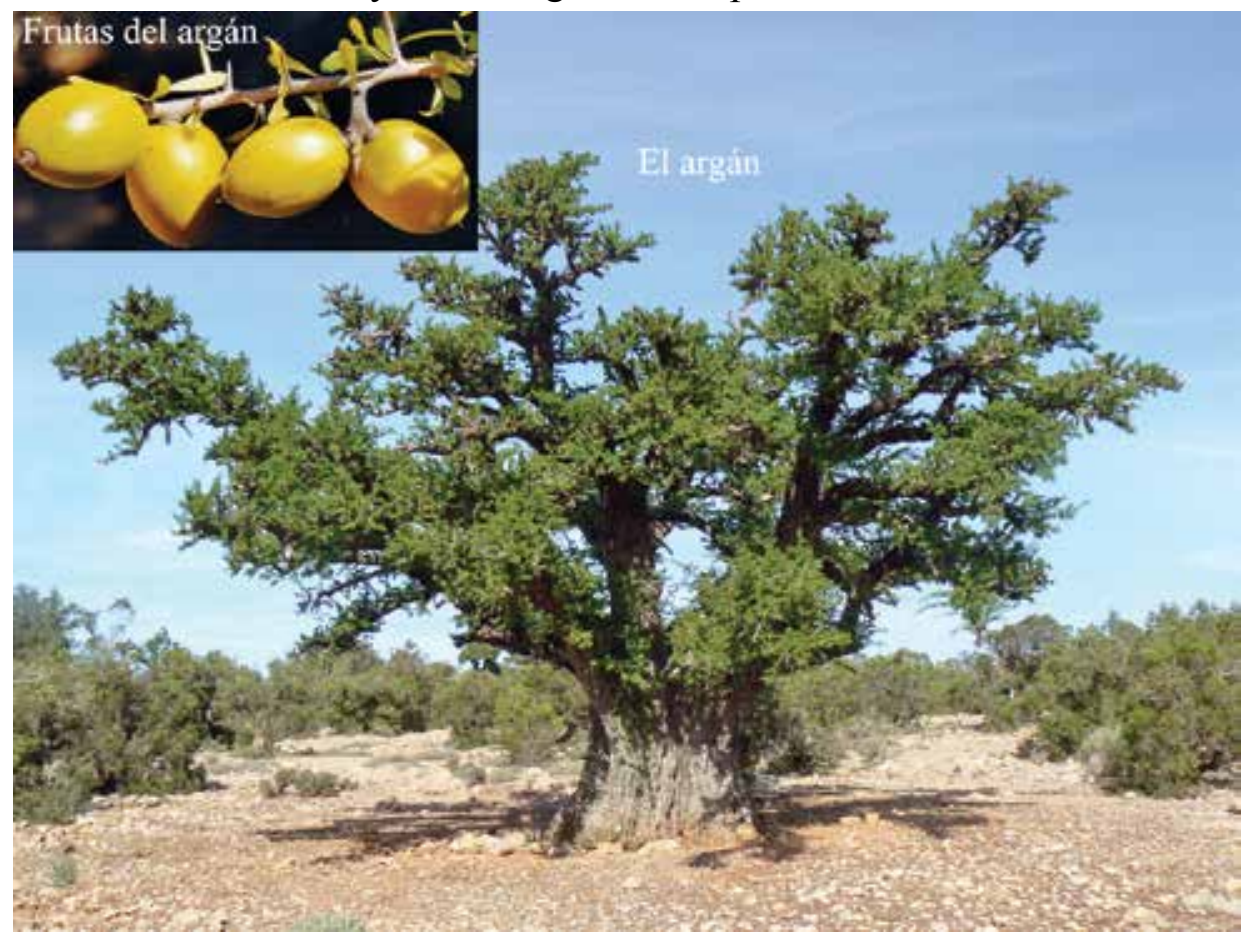

Fuente: Msanda, 2011. 
Figura 2. El área geográfica del argán

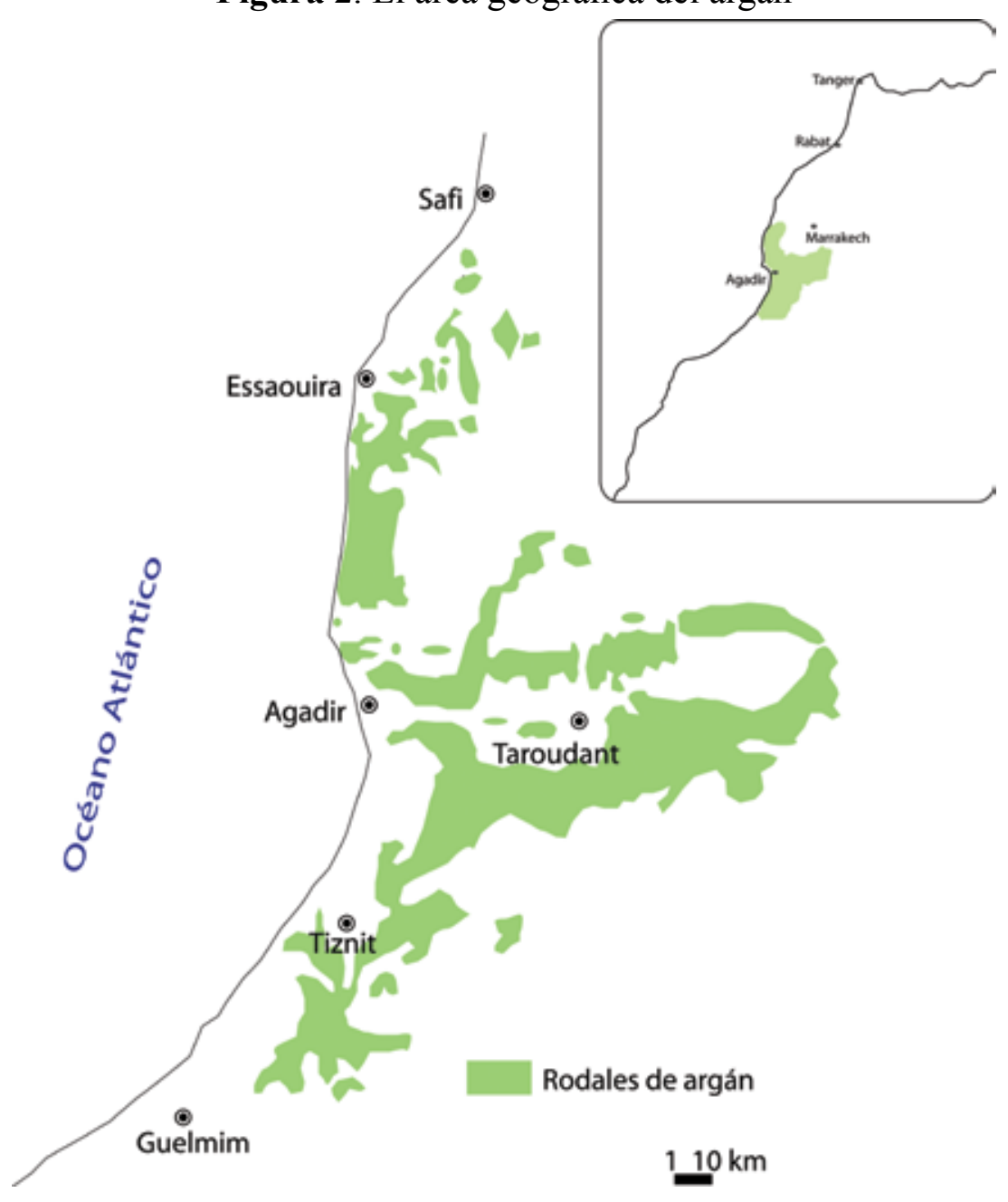

Fuente: Faouzi según M’hirit et al., 1998.

La degradación de los recursos naturales es un fenómeno muy antiguo en la región mediterránea (Boudy, 1952; McGregor y al., 2009). Las sociedades regularmente transforman y dan forma al paisaje para responder a sus necesidades y sus servicios (Wyman y Stein, 2010). Estas transformaciones, impuestas por el sistema operativo inapropiado de los ecosistemas naturales, son el origen de estados irreversibles de degradación (M'hirit y al., 1998; Benabid, 2000).

202 Revista Geográfica de América Central No 55 ISSN 1011-484X, julio-diciembre 2015, pp. 199-222 
El bosque de argán (Argania spinosa), también llamado arganería, forma parte de la zona de clima semiárido mediterráneo en el sudoeste de Marruecos en transición hacia Sáhara. Declarada Reserva de Biosfera (MAB, Man And Biosphere) por la UNESCO en 1998, constituye un sistema complejo susceptible de enriquecer las discusiones sobre los mecanismos de la dualidad factores/consecuencias de degradación y propiciar lecciones sobre la relación hombre-entorno (Elwahidi y al, 2014).

Los trabajos recientes distinguen la arganería de llanura de la arganería de montaña, dónde las condiciones ecológicas y económicas se diferencian sensiblemente. La Arganería de llanura parece muy amenazada, porque posee un gran potencial agrícola y absorbe a la mayoría de la emigración de las zonas de montaña, la evolución socioeconómica y la modernización de las explotaciones agrícolas desde los años 80 tienen como consecuencia el retroceso (distancia) de la cubierta forestal del 2,6\% (Elwahidi y al, 2014). En la arganería de montaña, dónde la población usuaria conserva un modo tradicional de utilización, se distinguen varias tendencias.

En efecto, las arganerías de las mesetas de Haha (Faouzi, 2003; McGregor y al., 2009), las laderas norte del Anti Atlas (El Aboudi, 2000) y de la región de Ait Baamrane (Genin y Simenel, 2011) siguen una dinámica débil de degradación. En la región de Awlouz, la provincia de Taroudan, Polain de Waroux y Lambin (2012) registraron una pérdida del 44,5\% en densidad del argán, las principales causas son el crecimiento de la aridez, el desarrollo del comercio de la leña utilizada como combustible y en una menor medida el pastoreo intensivo. Esta dinámica de degradación queda hasta hoy científicamente poco demostrada, lo que permite el desarrollo de discusiones generales alrededor de las dinámicas de degradación para el conjunto de la arganería.

El modelo de la arganería permite comprender por una parte la dinámica interactiva entre medio natural y medio socioeconómico y, por otra parte, someter a examen la hipótesis de que la degradación esté vinculada, la mayoría de las veces, a una sobreexplotación de los recursos por las poblaciones locales que se caracterizan por un fuerte crecimiento demográfico y un desarrollo económico débil (McGregor y al., 2009).

Ciertos autores sugieren no generalizar este modelo de degradación en toda la arganería marroquí (Genin y Simenel, 2011), bajo pretexto que las arganerías siguen diferentes ritmos de evolución, según la intensidad 
de las acciones antropológicas y de las condiciones climáticas. Nuestra investigación, intentará mostrar que con la ascensión comercial del aceite de argán, las prácticas que llevan a la degradación de la arganería tienden a generalizarse a todas las zonas de argán.

\section{EI desarrollo sostenible de la arganería}

El aceite de argán, producido a partir de la almendra oleaginosa (figura 2) fruto de la Argania spinosa (figura 3), es sin duda la producción en la que podría basarse un proyecto socio-económico. Gracias a sus propiedades el aceite de argán ha atraído la atención de los científicos, turistas, empresarios y grandes firmas de cosmética en todo el mundo, de todo esto ha resultado su fuerte demanda y el desarrollo de un mercado de alto valor del aceite de argán.

Figura 3. Semillas de argán (tiznine)

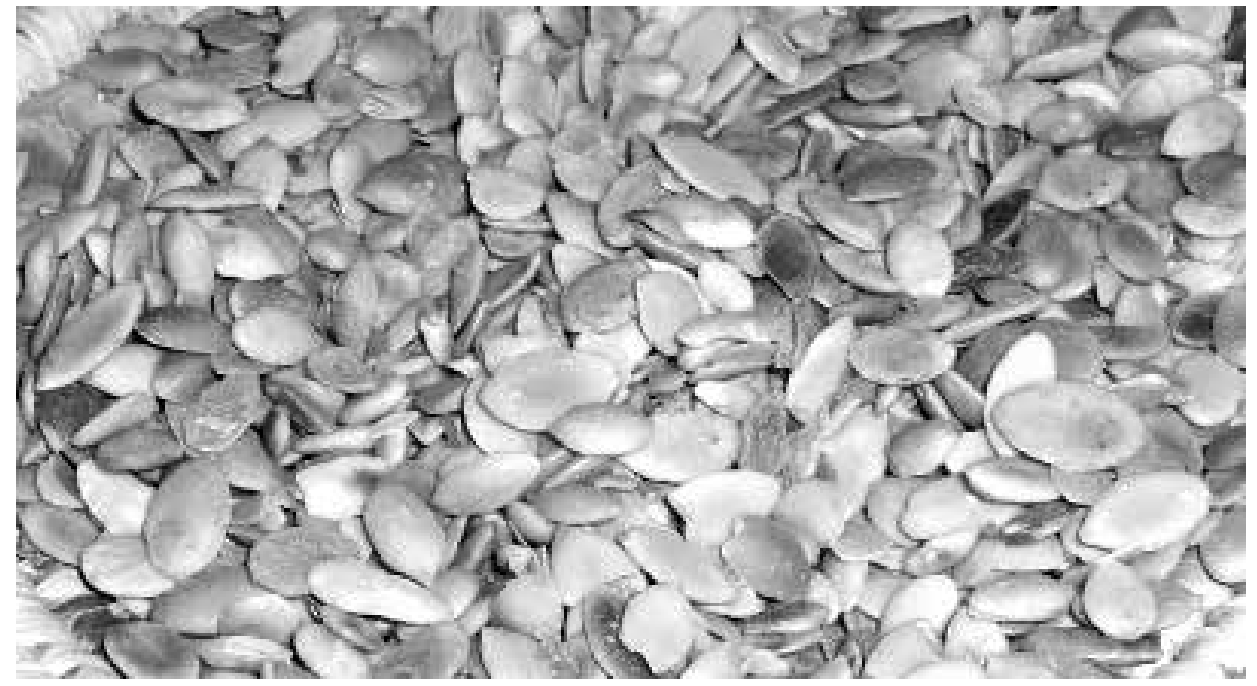

Fuente: Faouzi, 2010. 
Figura 4. Fruto del argán (akka)

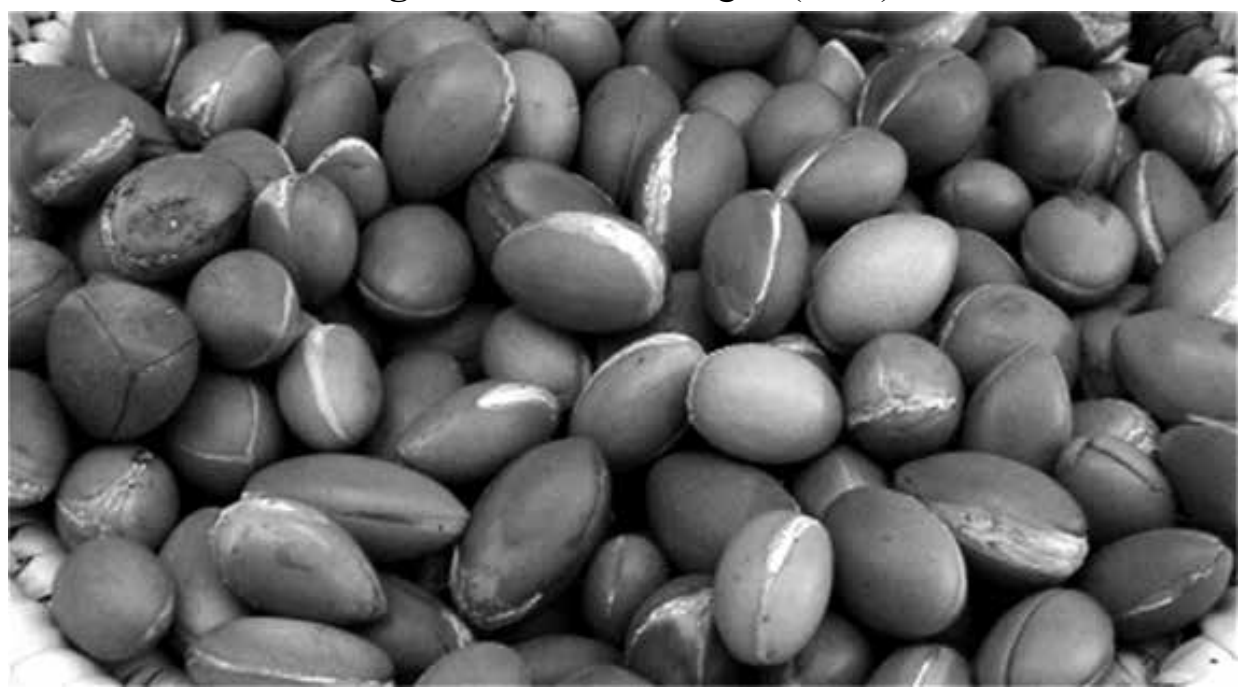

Fuente: Faouzi, 2010

Durante la década de 1990, este mercado de alto valor del aceite de argán, ha ido acompañado por esfuerzos de conservación y desarrollo a través de la creación de cooperativas de mujeres productoras de aceite de argán, que pasó de unos pocos cientos de mujeres en 1999, a más de 4000 en 2012. Estas cooperativas están organizadas en sindicatos o asociaciones de cooperativas de mujeres productoras de argán (UCFA) y dentro de los grupos de interés económico (GIE). En 2008, se creó AMIGHA, que incluye a todos los socios de la cadena del aceite de argán, su misión es la de reconocer y poner en práctica la Indicación Geográfica Protegida (IGP) de aceite de argán.

En otras palabras, favoreciendo la comercialización de aceite de argán en el mercado internacional, las mujeres pobres pueden aumentar sus ingresos (rol productivo) y pueden insertarse en grupos/cooperativas de mujeres (el papel de la comunidad), serán capaces de garantizar el bienestar de sus hijos y sus familias (función reproductiva). De esta manera, la importancia económica de estos recursos naturales afectará a la preservación del árbol de argán.

La apuesta de la gestión participativa y del desarrollo del sector económico de la arganería, es permitir la reducción de la extrema pobreza 
promoviendo la protección del árbol de argán. Este árbol, constituye una fuente de rentas suplementarias para las poblaciones rurales que, en su mayoría, se dedican a la agricultura. Los diferentes sectores de actividad de la arganería se hicieron una oportunidad de desarrollo, particularmente, a favor de las mujeres.

Queda, sin embargo, verificar que la distribución de la plusvalía es equitativa; es decir, que una parte suficiente de las rentas producidas por el aceite realmente vuelva a los usuarios de la arganería, con el fin de que esto beneficie la protección del medio (Chaussod y al., 2005). No obstante, el aceite de argán, uno de los principales productos de la arganería, dejaría de ser el principal factor de regresión de las superficies arboladas.

- El aceite de argán, un éxito comercial innegable

Ante el entusiasmo en torno a los productos basados en el aceite de argán, Marruecos (productor casi exclusivo), tiene que aprender rápidamente la mejor manera, así como las empresas internacionales que los comercialicen. Gracias a este monopolio, se deberían asegurar las exportaciones para generar una situación de ingresos así como fijar unos precios que le sean ventajosos. El precio de oro de los productos de argán desde la década de 1990 no ha dejado de aumentar (figura 5). La producción de este aceite se convierte en una gran ayuda para los índices de desarrollo económico del país. 
Figura 5. Evolución real de precios de los productos de argán en la provincia de Essaouira

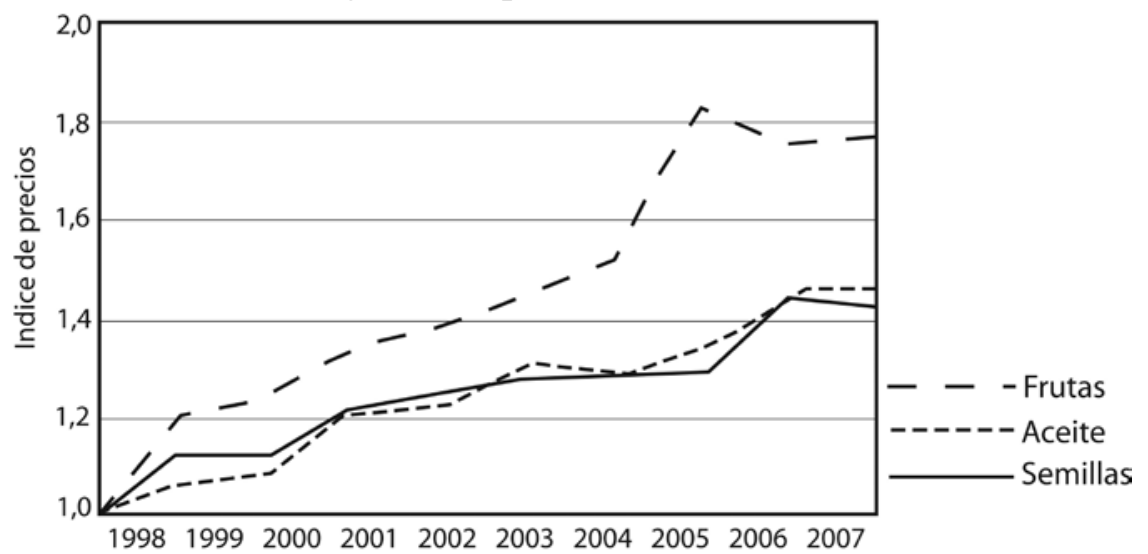

Fuente: Aboudrare et al., 2009.

Se sabe que en los últimos quince años, el aceite de argán es cada vez más popular entre las empresas y los consumidores occidentales de productos cosméticos. Según cifras oficiales, las cantidades de aceite de argán que se exportan han pasado a ser de 40 toneladas en 2003-2004 a más de 300 toneladas en el período 2007-2008, los principales países importadores son Francia (78\%), Alemania (7,6\%) y Suiza (7\%). Este éxito comercial genera indudables efectos positivos a nivel local: estructuración de los sectores, mejor calidad del producto, se ha reforzado la capacidad de producción y comercialización de las empresas marroquíes, la valoración del conocimiento local, los precios crecientes del aceite, la alfabetización de las mujeres productoras, etc.

Por el lado de las exportaciones, la locura de Europa, Estados Unidos y Japón por el aceite de argán ha provocado la aparición de un nuevo mercado para los productores y los sectores de exportación. Según las estadísticas del Organismo Autónomo de control y de la Coordinación de las Exportaciones (EACCE), las exportaciones de aceite de argán continuaron aumentando, hasta 343 toneladas en el período 2009-2010. Sin embargo, otros impactos más problemáticos acompañan esta "historia de éxito" y cuestionan los principios del desarrollo sostenible.

A pesar de este éxito comercial, las ganancias por el valor económico de aceite de argán no parecen beneficiar a la gente, especialmente a los 
pueblos bereberes, que son los productores primarios. Hay incluso otro fenómeno preocupante: el árbol de argán, en el que crece el fruto del que se extrae el aceite, tiende a una rápida disminución en el tamaño de su fruto y la densidad de plantas por superficie. Este viejo árbol, de 80 millones de años, está muy amenazado, cada año, cerca de 600 hectáreas de arganias desaparecen a pesar de su excelente resistencia a la sequía, la densidad del árbol de argán se reducirá en dos tercios en cincuenta años.

De ahí la necesidad de cuestionar la supervivencia de la arganería, ¿verán las próximas generaciones los bosques de argán en las laderas de las montañas del Atlas? ¿Podrán aspirar a un mejor nivel de vida que sus padres gracias a estos árboles? Mediante este artículo, vamos a tratar de responder a estas preguntas a través de un análisis que se basa en dos enfoques complementarios: el primero, es un gran trabajo de investigación de campo y el segundo, se basa en la documentación y una gran cantidad de consultas bibliográficas.

\section{Análisis ecológico de la degradación del ecosistema Argania spinosa}

Se observa que hasta la fecha, la reducción de la superficie del árbol de argán marroquí es grande y tiende a acelerar, aunque los datos disponibles en cuanto a la exactitud de los bosques del árbol de argán son limitados, si tomamos el ejemplo de la región Haha (Alto Atlas occidental), se pierden alrededor de 600 hectáreas por año (Bouzemouri, 2007), una reducción de dos tercios de la densidad de los bosques dentro de cincuenta años (figura 6); también se estima que la cubierta forestal en la región de Souss disminuyó en aproximadamente un 2,6\% entre 1989 y 2006 (Zugmeyer, 2006). Por último, la densidad media de cepas de argán inicialmente compuesta por 100 cepas/ha, ha pasado a 30 cepas/ha (Zugmeyer, 2006). 
http://dx.doi.org/10.15359/rgac.2-55.8

Hassan FAOUZI. Impacto de la evolucion del mercado del aceite de argán sobre la arganería de Marruecos

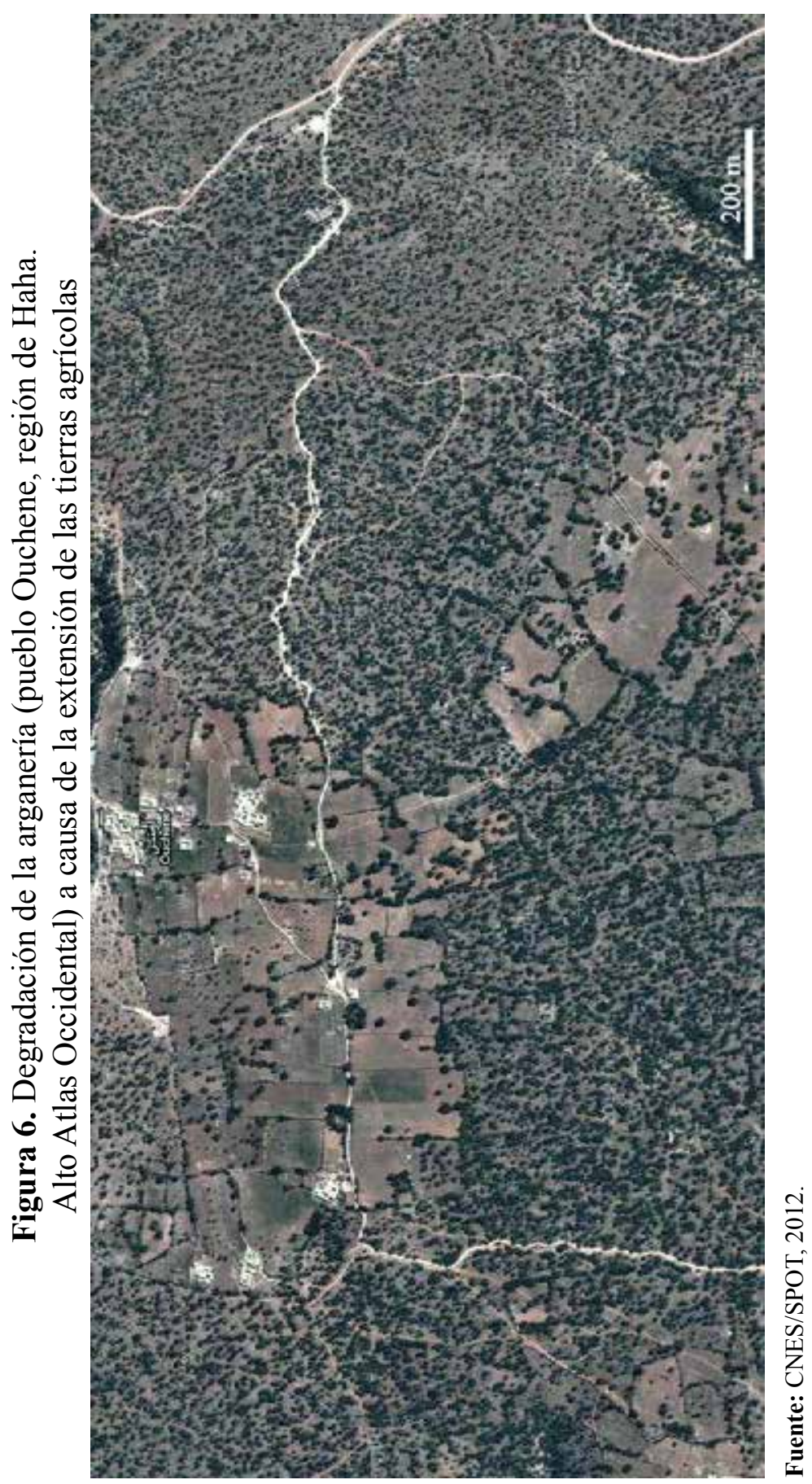

Revista Geográfica de América Central No 55

ISSN 1011-484X, julio-diciembre 2015, pp. 199-222 209

@) 
El éxito comercial de aceite de argán, va a propiciar aún más la degradación, aumentando la presión sobre el árbol de argán mediante la intensificación de la recogida de fruta, que no deja de tener consecuencias para la regeneración del árbol de argán.

\section{Una regeneración a merced de la producción}

La producción intensiva de argán está limitada por su carácter endémico. Por otro lado, en el plano económico, el aumento de los precios de exportación (multiplicado por 6 en 15 años) convirtió un mercado interno en un mercado de alquiler.

Los ecosistemas vegetales, se espera que sean capaces de reponerse de forma autónoma, una semilla es el origen de un árbol, esto se conoce como regeneración natural. El ciclo normal de crecimiento, desarrollo y degeneración, permite a las plantas nuevas ocupar el lugar de los árboles muertos de forma natural por su edad, un evento climático o causas parasitarias. La regeneración natural, es la capacidad de un ecosistema para recuperarse sin la intervención externa, especialmente, después de la destrucción parcial o total de un conjunto.

En la década de 1990, la situación ya era bastante pesimista: El árbol de argán no regeneraba naturalmente a partir de semillas (excepto en muy pocos casos) o plantaciones artificiales; la regeneración mediante siembra natural bajo el árbol no se observa en la mayor parte del territorio. Hoy en día, en el bosque, no ha habido casi ninguna plántula nacida de forma natural (Bellefontaine, 2010), salvo de manera excepcional al borde de las ramblas o en lugares muy protegidos (figuras 7 y 8 ). 
Figura 7. Plántulas de argán

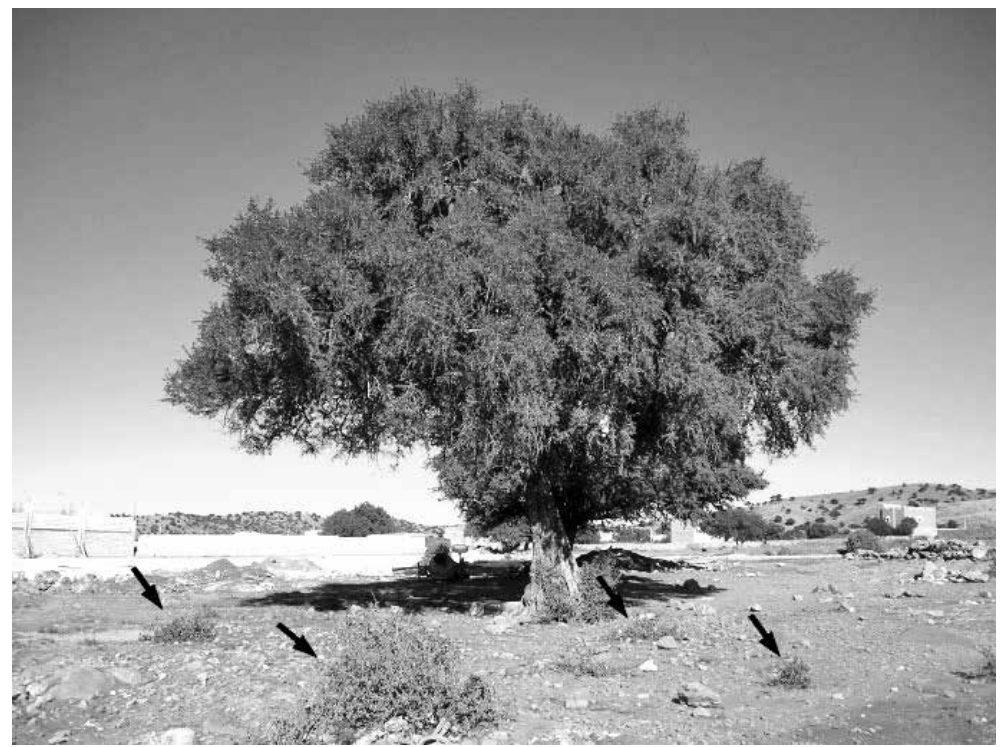

Fuente: Faouzi, enero 2013.

Figura 8. Arganería muy degradada debido a la ausencia de regeneración de argán

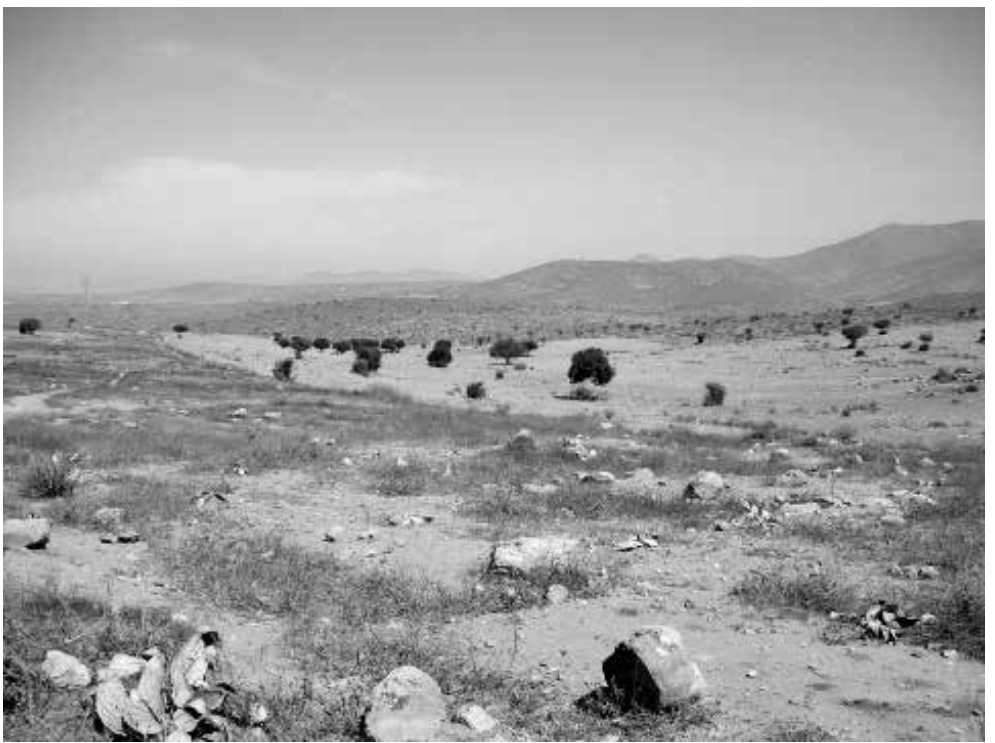

Fuente: Faouzi, mayo 2010. 
Hemos sido capaces de poner en relieve otra de las causas de la difícil regeneración natural de la arganería, en un principio, no parece haber un vínculo relevante entre la explotación de los frutos de este árbol para la producción de aceite de argán y la desforestación; después de todo, la vida de un árbol no está condicionada por el uso de sus frutos; pero pensemos que la semilla que hay dentro de la fruta es esencial para la reproducción del árbol ya que es esta la que germina para formar la nueva planta; sin embargo, según Bouzemouri (2007), la producción de aceite de argán moviliza a casi todos los frutos disponibles, evitando que las semillas caigan y germinen (figura 9) .

De hecho, las personas toman los frutos de los árboles ya sea de manera directa o buscando las almendras oleaginosas que no han podido digerir las cabras. En otras palabras, la probabilidad de que haya semillas que puedan germinar un nuevo árbol es baja. Los árboles están sobrexplotados ${ }^{2}$, este fenómeno está directamente relacionado con el éxito creciente de aceite de argán.

Figura 9. Bereberes marroquíes recogen los frutos de argán (affiache)

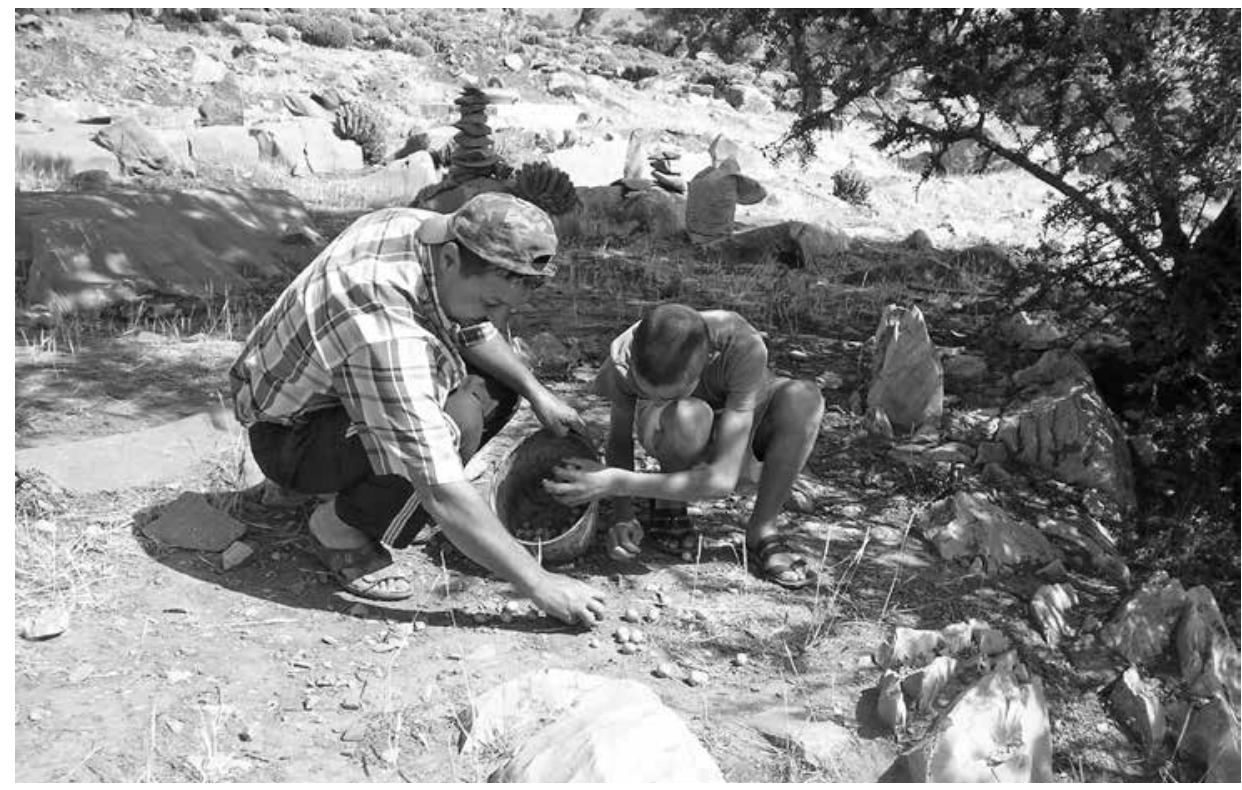

Fuente: Faouzi, 2014.

2 Vale la pena recordar que la producción de fruta (frutos secos Argán) varía dependiendo de la edad y la densidad (20 a $100 \mathrm{~kg} /$ árbol) con un promedio de $40 \mathrm{~kg}$ / árbol/año. Con base en la densidad media de argán (alrededor de 50 árboles por hectárea), la producción potencial se estima en 32000 toneladas de aceite de argán al año.

212 Revista Geográfica de América Central No 55 ISSN 1011-484X, julio-diciembre 2015, pp. 199-222 


\section{El aceite de argán: crecimiento de la demanda}

El crecimiento en la demanda de aceite, como un producto terminado o materia prima, ha continuado acelerándose en los últimos años, trayendo consigo un aumento continuo de las cantidades a suministrar; hay un aumento en la demanda de almendras que se venden como materia prima para la producción de aceite de argán (figura 10), por último, los granos contenidos en la fruta también se venden en los zocos. Las personas que poseen árboles de argán en sus tierras ven en este árbol una fuente adicional de ingresos, como consecuencia de todo esto cada vez surgen más intermediarios en la venta de este producto.

Figura 10. Gran stock de frutos de argán

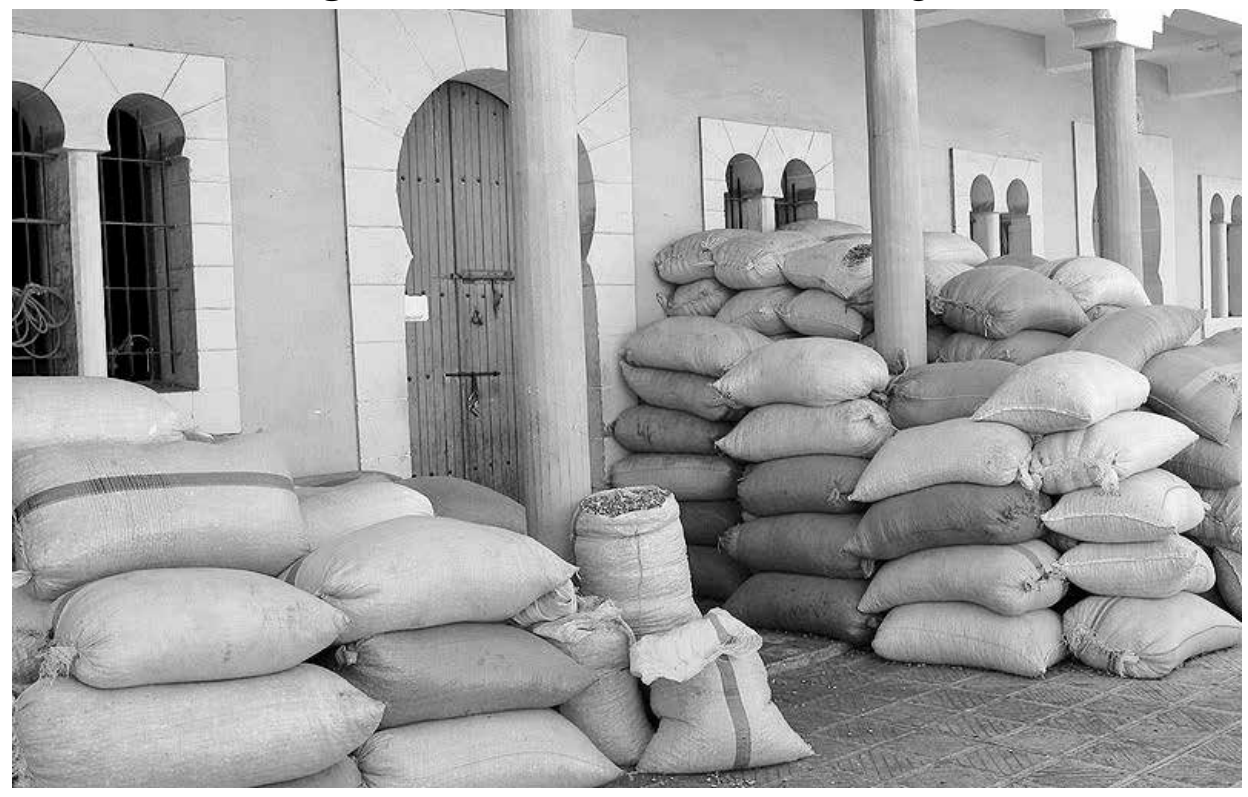

Fuente: D. Daniel, 2005.

La demanda de almendras oleaginosas se ha intensificado, no solo a nivel local sino nacional e internacional, para estas nuevas formas de producción, tiene que haber una oferta de producto capaz de satisfacer la creciente demanda; pero esto ha llevado a que hoy, todos los frutos de todos los árboles son recogidos, cortando así el ciclo de reproducción. En cuanto a los propios productores, la sobrexplotación de los árboles supone 
un problema en términos de productividad, las arganias cada vez mas explotadas, tienen rendimientos cada vez más bajos.

Tradicionalmente, el aceite de argán se movía en un espacio íntimo y familiar (Javiot, 2007), se encontraba también, ocasionalmente, en los zocos. Entre 1998 y 2007, el mercado de argán ha conocido una evolución espectacular. Hoy una decena de empresas comercializan esencialmente la producción con una exportación significativa, los precios de venta suben mucho en comparación con los mercados locales. La apertura del mercado a nivel nacional e internacional, propició un aumento repentino en los precios del aceite y de las semillas, hace 10 años un litro de aceite de argán valía 60 dirhams, mientras que hoy cuesta entre 160 y 200 dirhams, los precios de la fruta de argán (affiache) han seguido la misma tendencia que el aceite y, han conocido un aumento sustancial en razón de la fuerte demanda de importantes sociedades privadas que operan en los grandes zocos semanales como intermediarios; el precio de las semillas de argán se ha doblado, ha aumentado más rápidamente que el aceite.

La fuerte demanda de aceite de argán también conduce a una diferenciación de este en los mercados. De hecho, hay dos tipos de aceite de argán, uno de uso culinario, extraído en caliente después de tostar las semillas, y el otro de uso cosmético preparado en frío, generalmente, con la ayuda de prensas; el aceite de uso culinario es actualmente comercializado tanto en Marruecos como en el mundo entero. Sus precios varían enormemente de 200 dirhans por litro en los zocos a más de veinte veces este precio en selectos restaurantes de París o Nueva York, el mercado de aceite de argán también se disparó durante la última década, después de haberse confirmado la importancia de las propiedades químicas de este aceite. Los precios del aceite de uso cosmético sobrepasan en mucho los de aceite de uso culinario, llegando a los 50 dirhams los 50 mililitros (Aboudrare y al., 2009).

Por supuesto, la población local no puede permanecer indiferente a las ganancias derivadas de la subida de los precios del aceite (Simenel, 2009). Cada vez más familias comenzaron a vender el aceite de argán a través de las redes comerciales del zoco.

\section{Impacto de esta evolución}

En términos de producción, almacenamiento, venta y consumo de argán, después de una investigación realizada por Aboudrare et al. (2009),

214 Revista Geográfica de América Central No 55 ISSN 1011-484X, julio-diciembre 2015, pp. 199-222 
observamos que la producción de aceite de argán, en los hogares, fue casi tres veces mayor en 2007 que en 1999. La cantidad de frutos, recogida por familia, fue superior en la primera década del siglo XXI que en la última del siglo XX. El número de familias recogiendo frutas en las arganerías se ha más que duplicado a partir del año 2000, ahora se tiende a recoger los frutos manualmente en lugar de dejar que las cabras los coman directamente del árbol.

El cambio más dramático es el almacenamiento por parte de las familias del aceite y la fruta, se tiende a almacenar más aceite de argán y consumir casi la mitad menos que antes. El almacenamiento de la fruta, por su parte, casi se ha triplicado entre $1999(207 \mathrm{~kg})$ y $2007(581 \mathrm{~kg})$ (Aboudrare y al., 2009). El fruto de la Argania es ahora un componente importante del ingreso familiar y una especie de cuenta bancaria debido a su alto valor.

El resurgimiento del interés económico del aceite de argán, por parte de la población local ha tenido un impacto sobre las prácticas de explotación de la arganería, pero no en el sentido esperado, sino que ha originado un cierto trastorno del sistema tradicional, en la extensión territorial de las áreas de recolección.

En realidad, para la recogida de almendras, las mujeres no se conforman con las arganias situadas en sus campos, están cosechando los frutos por todo el bosque de argán; todos los frutos son recogidos, con la evolución del valor del los frutos y las exigencias de calidad, la mayor parte es recolectada manualmente ya que las almendras recolectadas a través de las cabras son de menos calidad (Aboudare y al., 2009). Incluso vemos personas que varean el árbol, lo que afecta a la próxima floración y pone en peligro la regeneración de esta planta. Las mujeres se comportan como depredadoras de los bosques de arganias y practican una recolección furtiva, una recolección que no concuerda con las prácticas de mantenimiento del árbol, por lo que están prohibidas en los bosques propiedad del estado. Este trabajo lo hacían tradicionalmente los hombres; pero, la mayoría emigraron a Europa o a las grandes ciudades de Marruecos en busca de trabajo.

La creciente demanda de aceite de argán para satisfacer el mercado nacional e internacional y el aumento de los precios de los productos de argán (aceite y granos), ha tenido efectos indirectos como la aparición de una serie de prácticas de recolección furtiva y la pérdida de prácticas 
productivas. Las semillas de argán (tiznine) de la misma especie de almendras $(a k k a)$ reflejan la falta de atención a los árboles. De un sistema de domesticación del árbol y el bosque en el que las prácticas agrícolas, ganaderas y forestales están estrechamente relacionadas, pasamos a un sistema de aniquilación donde solo se practica la recolección furtiva, las familias que viven en la pobreza, buscarán cada día beneficiarse de esta bonanza, recogiendo toda la fruta de argán (affiache) que encuentren en los bosques para venderla a los intermediarios.

El tamaño de los rebaños de cabras, es otro factor de degradación de la arganería, no han dejado de aumentar con fluctuaciones anuales relacionadas con variabilidad de las precipitaciones, desde finales de 1990. Esto indica que el aumento de los precios de argán no ha resultado en una reducción de la presión sobre el árbol a través de la disminución en el número de rebaños de cabras. Las familias que se han beneficiado del crecimiento del mercado de argán, son las que recogen más frutos, estas familias invierten más en la cría de cabras; este hecho parece incompatible con la teoría de la conservación, porque los ingresos de los productos forestales de argán se convierten en una amenaza para estos por el impacto negativo que las cabras representan para los árboles de argán (figura 11).

Figura 11. Cabras comiendo los frutos de argán

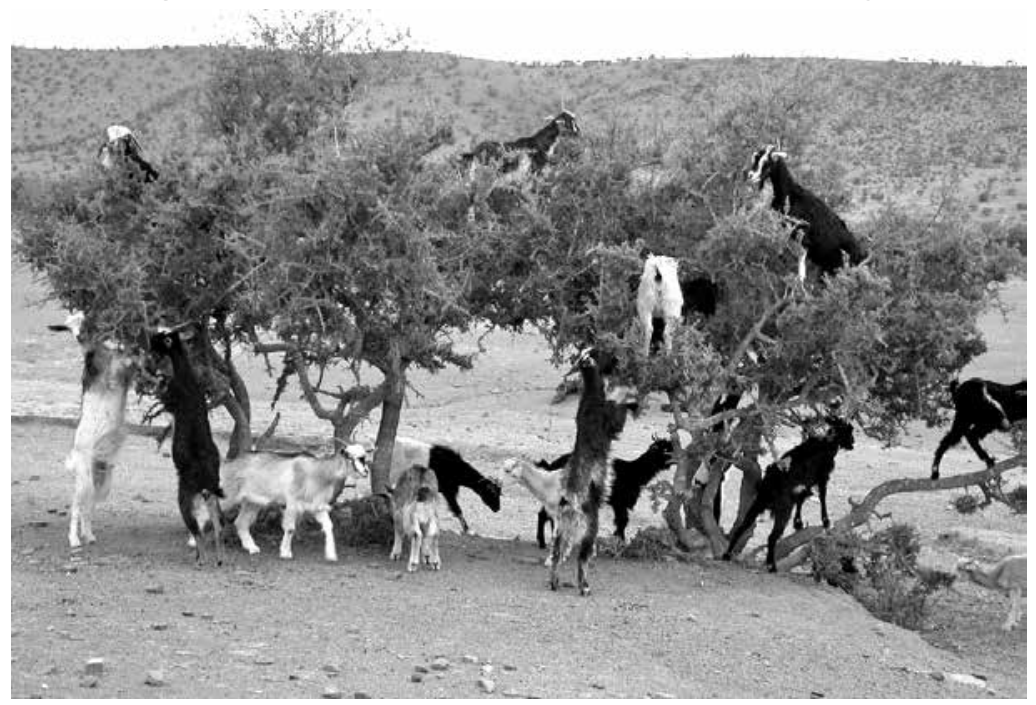

Fuente: J.P. Peltier. Goats climb the argan tree to eat its fruits (affiache).

216 Revista Geográfica de América Central No 55 ISSN 1011-484X, julio-diciembre 2015, pp. 199-222 
El auge del mercado de argán también ha dado lugar a más conflictos entre las personas que recolectan los frutos de la argania; los recolectores se han vuelto más agresivos en la recogida de la fruta a través de la utilización del vareo que puede causar un daño significativo en el árbol. Esta práctica, que consiste en golpear los árboles con palos o piedras para hacer caer la fruta antes de madurar, es el resultado de la multiplicación de los actos de robo de frutos en el bosque de argán (Aboudrare y al., 2009). La recolección de frutas, además de los efectos combinados de la sequía, crecimiento de la población y la pobreza, acentúa la degradación del árbol de argán. Cabe señalar que desde le principio de los años 2000, el costo de vida en Marruecos se ha incrementado en casi un $25 \%$, lo que afecta a la economía de las familias, como resultado los frutos de la Argania, son cada vez más apreciados y buscados agravando aún más la presión sobre el árbol.

El modelo cooperativo es ciertamente pertinente como medio de integración de las mujeres en el desarrollo, en la lucha contra la pobreza y por el mejoramiento de su calidad de vida de modo sostenible. Sobre el plan social, la cooperativa permite valorizar los recursos humanos mediante la formación y la educación, y pretende, del mismo modo garantizar el bienestar social y económico integrando a las personas marginadas con el fin de reducir la pobreza (Elkandoussi y al., 2011). Pero el sector "aceite de argane" que es muy lucrativo, ha atraído a otros numerosos operadores privados.

Ciertas sociedades privadas se hacen pasar por cooperativas y adoptan este estatuto para beneficiarse de sus ventajas fiscales y su imagen. Sobre las 150 cooperativas que existen hoy, una treintena, solamente, tiene un funcionamiento verdadero y cooperativo. Diferentes intermediarios especulan con la materia prima. En pocos años atrás, mientras que los intermediarios se multiplicaban, industriales marroquíes y europeos instalaron en Casablanca y en Marrakech pequeñas fábricas equipadas con máquinas de extracción capaces de extraer el producto según las normas de la exportación.

Ciertas empresas van a transformar el almidón, directamente, en Europa, a pesar de la ilegalidad de esta práctica. Por encima de la producción, cooperativas e industriales entran en competencia para abastecerse de materia prima (affiache), la cual puede faltar algunos años debido a la sequía. Los industriales se abastecen a través de intermediarios diversos, desde recolectores a mayoristas. 
Por todas partes, los especuladores se hacen con las existencias de frutas para revenderlas a precios altos a los industriales, obligados a pagar la cuantía que se les pide. En consecuencia, varias "cooperativas dejan de producir por falta de fondos para abastecerse de frutas" (Raimbeau, 2009). Ante esta situación, nos preguntamos si con la búsqueda del "rendimiento" en el medio plazo, no estamos oficializando la reproducción del desierto por las destrucciones de las últimas murallas naturales que lo retrasan.

Es urgente pensar en una utilización sostenible de este recurso como vía que hay que privilegiar para asegurar su protección (Boisvert y Vivien, 2007). Esto pasa por la adopción de un enfoque de desarrollo global e integrado que haga participar a todos los actores locales. Principalmente, se trata de pensar en una gestión de la arganería que debe asociar a responsables, científicos, expertos y poblaciones locales en acciones comunes (Pinton y Grenand, 2007), y también, por una convivencia entre el saber científico y el saber tradicional.

Es verdad que el número de acciones que van en esta dirección vieron la luz (creación de asociaciones de usuarios, creación de la Agencia Nacional de Desarrollo de las Zonas de los Oasis y de la Arganería (ANDZOA). Por el contrario, estas acciones les falta armonía, porque son llevadas de manera individual y sin ninguna coordinación. Entonces, se debe realizar una reforma en profundidad de la política forestal que reconozca los colectivos y los usuarios, identificar derechos (colectivos o privados) bajo una gestión realmente concertada (Bourbouze y El Aïch, 2005).

Si el estatuto colectivo de la arganería constituye una de las mejores garantías actuales contra una acentuación del proceso de degradación corriente, pero, sigue siendo la causa principal de numerosos conflictos y no está adaptado a la situación actual. La gestión de estos conflictos, es una necesidad para garantizar el desarrollo local.

En un contexto marcado por el crecimiento demográfico, la sequía y por un nivel de pobreza muy elevado ${ }^{3}$ (Faouzi, 2009), la evolución económica del sector del aceite de argán, afectan el equilibrio ecológico de la arganería, cuya perennidad está puesta en peligro por una explotación superior a las capacidades de producción (Nouaim, 2005).

3 Según el censo general de la población y del hábitat 2004 (HCP, Marruecos), más de $45 \%$ de la población de las zonas del argan vive con menos de 300 euros/año es decir menos de 30 euros/mes.

218 Revista Geográfica de América Central No 55 ISSN 1011-484X, julio-diciembre 2015, pp. 199-222 


\section{Conclusión}

A pesar de los esfuerzos de los recolectores para proteger sus árboles en propiedad, sus actitudes y comportamiento hacia el bosque, no muestra un interés por la conservación. El auge del mercado de argán no ha propiciado un comportamiento colectivo de conservación para estos bosques. La evolución económica en el sector del aceite de argán, por lo tanto, representa una grave amenaza para la arganería marroquí.

El mayor desafío es cambiar la percepción de las personas con un enfoque de protección de los árboles a corto plazo para mejorar la producción de fruta, en una visión a más largo plazo para conservar la arganería marroquí. Se trataría de sensibilizar a la población por todos los medios (medios de comunicación, las mezquitas, el zoco, etc.) y, despertar su interés por esta cultura de preservación del patrimonio del bosque de argán en programas de formación en las escuelas de primaria y secundaria de la región de argán.

A plazo más o menos largo, si no se hace nada, todo el suroeste de Marruecos está amenazado por la desertización, debido a que el equilibrio del sistema es frágil y su sostenibilidad está amenazada por la explotación que supera la capacidad de producción.

El desarrollo y el crecimiento económico a la vez sostenible y duradero, sólo es posible si mejoramos la situación económica, social, política, jurídica y cultural de la zona. El desarrollo sostenible, también debe basarse en el desarrollo social equitativo, que da a los pobres los medios para explotar racionalmente los recursos ambientales.

El futuro de la argania, depende en gran medida del nivel de vida y oportunidades para promover actividades generadoras de ingresos que puedan proporcionar apoyo público para la preservación de este árbol, para asegurar una mejor regeneración del bosque de Arganias, se deben promover sistemas agroforestales que combinan varias especies; el nopal o la acacia espinosa, por ejemplo, se adaptan bien a la aridez local, al tiempo que tienen un crecimiento más rápido que el árbol de argán, esto proporcionaría productos adicionales y forraje de verano (Benchekroun y Buttoud, 1989). La Argania spinosa, se ha ganado el respeto del hombre y siempre ha proporcionado un importante papel socio-económico por sus múltiples usos (Ezzahiri y al. 2007). La desaparición de esta especie que determina la existencia rural del suroeste de Marruecos, tendrá graves 
consecuencias en los ámbitos económico, político, social y ambiental de la zona (Faouzi, 2012).

\section{Referencias}

Aboudrare, A., Lybbert, T. J. et Magnan, N. (2009). Le marché de l'huile d'argan et son impact sur les ménages et la forêt dans la région d'Essaouira, Enquête diagnostic, Transfert de technologie en agriculture, Bulletin Mensuel de Liaison et d'Information du PNTTA, 175.

Bellefontaine, R. (2010). De la domestication à l'amélioration variétale de l'arganier (Argania spinosa L. Skeels) ? Science et changements planétaires / Sécheresse, 21(1), 42-53.

Benabid A., (2000). Flore et écosytèmes du Maroc : évaluation et préservation de la biodiversité. Ibis Press, Paris, France, 359.

Benchekroun, F. et Buttoud, G. (1989). L'arganeraie dans l'économie rurale du sud-ouest marocain. Forêt Méditerranéenne, T. XI, 2.

Boudy, P. (1952). Guide forestier en Afrique du Nord. La Maison Rustique, Paris, France, 510.

Bouzemouri, B. (2007). «Problématique de la conservation et du développement de l'arganeraie ", dans Zoubida Charrouf (sous la dir. de): «L'arganier : levier du développement humain du milieu rural marocain», colloque international organisé par l'association Ibn Al Baytar à la faculté des sciences de Rabat.

Charrouf, Z. (2007). 20 ans de recherche-action pour faire de l'arganier un levier du développement durable du milieu rural marocain. Actes $d u$ colloque international «l'arganier un levier du développement humain du milieu rural marocain». Faculté des Sciences, Université Mohammed V-Agdal, Rabat, Maroc, 3-12.

De Waroux, YP., Lambin, EF. (2012). Monitoring degradation in arid and semiarid forests and woodlands: the case of the argan woodlands (Morocco). Applied Geography, 32 (2), 777-786.

El Aboudi, A. (2000). Télédétection et cartographie de la végétation en zone aride. Application à la terminaison occidentale de l'Anti-Atlas marocain. Thèse de doctorat d'état es-sciences biologiques Univ. Mohammed V, Rabat, Maroc, 159. 
Elwahidi, F., Bellefontaine, R., Quentin, P. et Defourny, P. (2014). Dynamique de changement de l'arganeraie entre sur-usage et mutations sociales: une opportunité d'équilibre socio-écologique? Journal of Agriculture and Environment for International Development JAEID 108 (2): 109-133. doi: 10.12895/jaeid.20142.210

Emberger, L. (1938). Les arbres du Maroc et comment les reconnaître. Larose, éditeurs, Paris, 318.

Ezzahiri, M., Belghazi, B., Charradi, N. (2007). Contribution de l'Arganier dans le Développement Local de la Région d'Essaouira. Annales de la recherche forestière au Maroc, 38, 209-210.

Faouzi, H. et Martin, J. (2014). Soutenabilité de l'arganeraie marocaine. Confins, 20. doi: 10.4000/confins. 8842

Faouzi, H. (2012). «Impact des coopératives féminines sur la préservation et la valorisation de l'arganeraie: cas de la coopérative Tafyoucht (confédération des Ait Baâmrane, Anti-Atlas, Maroc) ", Confins [Online], 14 | 2012, doi:10.4000/confins.7521

Faouzi, H. (2012). La arganería de haha, un sistema agrario en crisis (Alto Atlas Occidental, Marruecos). Revista Geográfica de América Central, 48, 163-177.

Faouzi, H. (2003). L'arganeraie des haha: étude d'un système agraire en mutation (Haut-Atlas occidental, Maroc), Thèse doctorat: géographie: université Nancy 2, 500.

Genin, D. and Simenel, R. (2011). Endogenous Berber Forest Management and the Functional Shaping of Rural Forests in Southern Morocco: Implications for Shared Forest Management Options. Human Ecology, 39(3), 257-269.

Lefhaili, A. (2010). FAO Forest Resources Assessment: Morocco Country. Report, Rome: FAO, 63.

Mcgregor, H.V., Dupont, L., Stuut, J.-B.W., Kuhlmann, H. (2009). Vegetation change, goats and religión: a 2000-year history of land use in southern Morocco. Quaternary Science Reviews, 28 (15-16), 1434-1448.

M'hirit, O., Benzyane, M., Benchekroun, F., El Yousfi, S.M., Bendaanoun, M. (1998). L'arganier, une espèce fruitière-forestière à usages multiples. Mardaga, Sprimont, Belgique, Belgique, 145. 
Simenel, R. (2009). L'argan: l'huile qui cache la forêt domestique. De la valorisation du produit à la naturalisation de l'écosystème. Autrepart, (50), 51-74.

Wyman, M.S. and Stein, T.V. (2010). Modeling social and land-use/landcover change data to assess drivers of smallholder deforestation in Belize. Applied Geography, 30 (3), 329-342.

Zugmeyer, L. (2006). Projet de développement sylvopastoral de l'arganeraie marocaine (commune rurale de Tiout, Taroudan, Ma$r o c)$. Mémoire de fin d'études, ENGREF, Nancy, France. 